\title{
Interrelationship between Seed Yield and Component Characters in Chickpea (Cicer arietinum L.)
}

\author{
Anmol Kerketta*, G.R. Lavanya and Promish Kumar
}

Department of Genetics and Plant Breeding, Sam Higginbottom University of Agriculture, Technology \& Sciences, Allahabad, India

*Corresponding author

\section{A B S T R A C T}

Keywords

Chickpea,

Correlation, Path coefficient

Article Info

Accepted:

04 June 2018

Available Online:

10 July 2018
Correlation and Path coefficient analysis were used to determine the relationship among yield and yield components done with 12 chickpea (Cicer arietinum L.) genotypes. The experiment was designed in Randomized Block Design with four replication. Biological yield, number of pods per plant, seed index and harvest index had shown positive significant correlation with the seed yield per plant. The positive direct effect on seed yield was revealed by biological yield followed by seed index. Therefore these traits can be taken into consideration while exercising selection for seed yield in chickpea.

\section{Introduction}

Chickpea $(2 \mathrm{n}=16)$ (Cicer arietinum $\mathrm{L}$.) with a genome size of $750 \mathrm{Mbp}$ is an ancient selfpollinated legume crop possibly originated in the fertile crescent region of southeastern Turkey and adjoining Syria and the third most important pulse crop grown in more than 44 countries worldwide van der Maesen (1987) and Arumuganathan and Earle (1991). It restore soil fertility through biological nitrogen fixation with the help of symbiotic bacteria Rhizobium in roots thus grown in rotation with other crops (Albayrak et al., 2006). Seed yield of chickpea is a complex character which is controlled by polygenes
(Thakur and Saini, 1995). Some of the characters such as biological yield, number of pods per plant, primary branches are highly correlated among themselves and with seed yield. The knowledge of inter relationship between different characters is important for determining selection criteria. Correlation studies provide better understanding of yield component (Robinson et al., 1951 and Johnson et al., 1955). Path coefficient analysis allows the partitioning of correlation coefficient into its components (Dewey and $\mathrm{Lu}, 1959)$. The present study was undertaken to elucidate the association between yield and its attributes in chickpea. 


\section{Materials and Methods}

The research experiment was carried out during rabi season, 2016-2017 comprising 12 genotypes of chickpea at the Field Experimentation Center of the Department of Genetics and Plant Breeding, Sam Higginbottom University of Agriculture, Technology and Sciences (Formerly Allahabad Agricultural Institute), Allahabad. Experimental material was obtained from Indian Institute of Pulse Research, Kanpur under AICRP on chickpea. The experiment was laid out in randomized block design with three replications. Plant to plant and row to row distance was maintained at $20 \mathrm{~cm}$ and 30 $\mathrm{cm}$, respectively. The recommended dose of fertilizer @ 20:40:40 N: P: K kg/ha was applied in the form of Urea and DiAmmonium Phosphate as basal dose at the time of sowing. It is not possible to record observations of each and every plant because of more number of genotypes and large population size. Hence, the technique of random sampling was adopted for recording the observations of various quantitative characters of chickpea. There plants of each treatment from each replication were selected at random at the time of recording the data on various characters. Readings from three plants were average replication-wise and mean data was used for statistical analysis, except days to $50 \%$ flowering and days to maturity. Data were recorded on Days to $50 \%$ flowering, Plant height $(\mathrm{cm})$, Number of primary branches per plant, Number of pods per plant, Days to maturity, Biological yield per plant (g), Harvest index (\%), Seed index (g), Seed yield per plant $(\mathrm{g})$. The data were subjected to association analysis as per method given by Al-Jibouri et al., (1958) and path analysis according to Dewey and Lu (1959).

\section{Results and Discussion}

Analysis of variance revealed that mean sum of squares due to genotypes here significant for all the characters. On the basis of mean performance $\mathrm{C}-127$ had maximum seed yield per plant (22.60) followed by C-136, C-128. High genotypic and phenotypic co efficient of variation was observed for biological yield. Pods per plant GCV (16.25) PCV (24.28) followed by seed index GCV (10.43) PCV (16.98) and harvest index GCV (7.93) PCV (11.65) indicating the presence of high amount of variation and role of environment on the expression of these characters. High genetic advance was depicted for biological yield 22.36 (Table 1-5).

Table.1 Estimates the genetic parameters for nine quantitative characters in chickpea genotypes

\begin{tabular}{|c|c|c|c|c|c|c|c|c|}
\hline \multirow[t]{2}{*}{ S.No. } & \multirow[t]{2}{*}{ Characters } & \multirow[t]{2}{*}{$\sigma^{2} g$} & \multirow[t]{2}{*}{$\sigma^{2} p$} & \multicolumn{2}{|c|}{ Coefficient of variation } & \multirow{2}{*}{$\begin{array}{c}\mathrm{h}^{2} \\
\text { (bs) } \%\end{array}$} & \multirow[t]{2}{*}{ GA } & \multirow{2}{*}{$\begin{array}{c}\text { GA as } \\
\% \text { of }\end{array}$} \\
\hline & & & & GCV & PCV & & & \\
\hline 1 & Days to $50 \%$ Flowering & 2.73 & 3.99 & 1.82 & 2.21 & 68 & 3.61 & 3.98 \\
\hline 2 & Plant Height & 52.24 & 56.72 & 13.02 & 13.56 & 92 & 18.31 & 32.98 \\
\hline 3 & Primary Branches/ Plant & 0.03 & 0.05 & 5.42 & 7.48 & 53 & 0.32 & 10.37 \\
\hline 4 & Pods/ Plant & 92.70 & 206.99 & 16.25 & 24.28 & 45 & 17.01 & 28.71 \\
\hline 5 & Days to Maturity & 1.53 & 4.13 & 1.04 & 1.71 & 37 & 1.99 & 1.67 \\
\hline 6 & Biological Yield & 91.63 & 117.09 & 26.80 & 30.29 & 78 & 22.36 & 62.59 \\
\hline 7 & Harvest Index & 13.92 & 30.06 & 7.93 & 11.65 & 46 & 6.70 & 14.24 \\
\hline 8 & Seed Index & 6.09 & 16.15 & 10.43 & 16.98 & 38 & 4.00 & 16.90 \\
\hline 9 & Seed Yield/ Plant & 17.20 & 22.39 & 25.00 & 28.53 & 77 & 9.60 & 57.85 \\
\hline
\end{tabular}

$\sigma^{2} \mathrm{~g}=$ Genotypic variance, $\sigma^{2} \mathrm{p}=$ Phenotypic variance, $\mathrm{GCV}=$ Genotypic coefficient of variance, PCV = Phenotypic coefficient of variance, $\mathrm{h}^{2}$ (bs) $\%=$ Heritability (broad sense), $\mathrm{GA}=$ Genetic advance, $\mathrm{GA}$ as $\%$ of mean = Genetic advance as percent of mean. 
Table.2 Estimates of genotypic correlation coefficient between yield and its components trait of chickpea

\begin{tabular}{|c|c|c|c|c|c|c|c|c|c|}
\hline S.No. & Character & $\begin{array}{l}\text { Plant } \\
\text { Height }\end{array}$ & $\begin{array}{l}\text { Primary } \\
\text { Branches/ } \\
\text { Plant }\end{array}$ & $\begin{array}{l}\text { Pods/ } \\
\text { Plant }\end{array}$ & $\begin{array}{l}\text { Days to } \\
\text { Maturity }\end{array}$ & $\begin{array}{l}\text { Biological } \\
\text { Yield }\end{array}$ & $\begin{array}{l}\text { Harvest } \\
\text { Index }\end{array}$ & Seed Index & $\begin{array}{l}\text { Seed } \\
\text { Yield/ } \\
\text { Plant }\end{array}$ \\
\hline 1 & Days to $50 \%$ Flowering & $-0.3795 * *$ & $0.2917 * *$ & 0.0131 & $0.4796 * *$ & 0.1113 & $0.2140 *$ & -0.0595 & 0.1714 \\
\hline 2 & Plant Height & 1.00 & $-0.4541 * *$ & -0.1788 & $-0.4289 * *$ & $-0.3204 * *$ & $-0.5532 * *$ & $-0.3769 * *$ & $-0.4457 * *$ \\
\hline 3 & Primary Branches/ Plant & & 1.00 & -0.0272 & $0.4710 * *$ & 0.1875 & 0.0076 & $0.4317 * *$ & $0.2293 *$ \\
\hline 4 & Pods/ Plant & & & 1.00 & $-0.7913 * *$ & $0.7677 * *$ & 0.1053 & $0.7199 * *$ & $0.8032 * *$ \\
\hline 5 & Days to Maturity & & & & 1.00 & $-0.3849 * *$ & $0.5879 * *$ & 0.1550 & $-0.2506 *$ \\
\hline 6 & Biological Yield & & & & & 1.00 & $-0.2306^{*}$ & $0.7881 * *$ & $0.9673 * *$ \\
\hline 7 & Harvest Index & & & & & & 1.00 & $0.0238 *$ & 0.0550 \\
\hline 8 & Seed Index & & & & & & & 1.00 & $0.8236 * *$ \\
\hline
\end{tabular}

$*$ and $* * 5 \%$ and $1 \%$ level of significance respectively

Table.3 Estimates of phenotypic correlation coefficient between yield and its related traits in 12 chickpea genotypes

\begin{tabular}{|c|c|c|c|c|c|c|c|c|c|}
\hline S.No. & Character & $\begin{array}{l}\text { Plant } \\
\text { Height } \\
(\mathrm{cm})\end{array}$ & $\begin{array}{l}\text { Primary } \\
\text { Branches/ } \\
\text { Plant }\end{array}$ & $\begin{array}{l}\text { Pods/ } \\
\text { Plant }\end{array}$ & $\begin{array}{l}\text { Days to } \\
\text { Maturity }\end{array}$ & $\begin{array}{l}\text { Biological } \\
\text { Yield (g) }\end{array}$ & $\begin{array}{l}\text { Harvest } \\
\text { Index }\end{array}$ & Index & $\begin{array}{l}\text { Seed Yield/ } \\
\text { Plant }(\mathrm{g})\end{array}$ \\
\hline 1 & Days to $50 \%$ Flowering & $-0.3023 * *$ & $0.2422 *$ & -0.1032 & $0.2350 *$ & 0.0359 & 0.1642 & 0.0556 & 0.0686 \\
\hline 2 & Plant Height & 1.00 & $-0.3062 * *$ & $-0.2198 *$ & $-0.2738 *$ & $-0.2701^{*}$ & $-0.3597 * *$ & $-0.2456^{*}$ & $-0.3888 * *$ \\
\hline 3 & $\begin{array}{l}\text { Primary Branches/ } \\
\text { Plant }\end{array}$ & & 1.00 & 0.0205 & 0.2068 & 0.1499 & 0.0159 & 0.1410 & 0.1310 \\
\hline 4 & Pods/ Plant & & & 1.00 & -0.0826 & $0.5174 * *$ & -0.0196 & $0.2417 *$ & $0.5535 * *$ \\
\hline 5 & Days to Maturity & & & & 1.00 & -0.0815 & 0.1164 & 0.1754 & -0.0644 \\
\hline 6 & Biological Yield & & & & & 1.00 & $-0.2323 *$ & $0.4855^{* *}$ & $0.9126 * *$ \\
\hline 7 & Harvest Index & & & & & & 1.00 & 0.1225 & 0.0953 \\
\hline 8 & Seed Index & & & & & & & 1.00 & $0.5181 * *$ \\
\hline
\end{tabular}

$*$ and $* * 5 \%$ and $1 \%$ level of significance respectively 
Table.4 Direct and indirect effect of yield component characters on seed yield in chickpea at genotypic level

\begin{tabular}{|c|c|c|c|c|c|c|c|c|c|c|}
\hline S.No. & Character & $\begin{array}{l}\text { Days to } \\
50 \% \\
\text { Flowering }\end{array}$ & $\begin{array}{l}\text { Plant } \\
\text { Height }\end{array}$ & $\begin{array}{l}\text { Primary } \\
\text { Branches/ } \\
\text { Plant }\end{array}$ & $\begin{array}{l}\text { Pods/ } \\
\text { Plant }\end{array}$ & $\begin{array}{l}\text { Days to } \\
\text { Maturity }\end{array}$ & $\begin{array}{l}\text { Biological } \\
\text { Yield }\end{array}$ & $\begin{array}{l}\text { Harvest } \\
\text { Index }\end{array}$ & Index & $\begin{array}{l}\text { Seed } \\
\text { Yield/ } \\
\text { Plant }\end{array}$ \\
\hline 1 & $\begin{array}{l}\text { Days to } 50 \% \\
\text { Flowering }\end{array}$ & -0.1014 & 0.0385 & -0.0296 & -0.0013 & -0.0486 & -0.0113 & -0.0217 & 0.0060 & 0.1714 \\
\hline 2 & Plant Height & -0.1049 & $0.2764 *$ & -0.1255 & -0.0494 & -0.1186 & -0.0886 & -0.1529 & -0.1042 & $-0.4457 * *$ \\
\hline 3 & $\begin{array}{l}\text { Primary Branches/ } \\
\text { Plant }\end{array}$ & 0.0596 & -0.0928 & 0.2044 & -0.0056 & 0.0963 & 0.0383 & 0.0015 & 0.0883 & $0.2293 *$ \\
\hline 4 & Pods/ Plant & -0.0008 & 0.0104 & 0.0016 & -0.0583 & 0.0462 & -0.0448 & -0.0061 & -0.0420 & $0.8032 * *$ \\
\hline 5 & Days to Maturity & 0.0259 & -0.0232 & 0.0255 & -0.0428 & 0.0540 & -0.0208 & 0.0318 & 0.0084 & $-0.2506^{*}$ \\
\hline 6 & Biological Yield & 0.1604 & $-0.4616^{* *}$ & $0.2702^{*}$ & $1.1061 * *$ & $-0.5545^{* *}$ & $1.4409 * *$ & $-0.3323 * *$ & $1.1356^{* *}$ & $0.9673 * *$ \\
\hline 7 & Harvest Index & 0.1159 & $-0.2995 * *$ & 0.0041 & 0.0570 & $0.3183 * *$ & -0.1248 & $0.5414 * *$ & 0.0129 & 0.0550 \\
\hline 8 & Seed Index & 0.0167 & 0.1060 & -0.1214 & -0.2025 & -0.0436 & -0.2217 & -0.0067 & $-0.2813 * *$ & $0.8236^{* *}$ \\
\hline
\end{tabular}

Residual effect $=1.03$

Table.5 Direct and indirect effect of yield component characters on seed yield in chickpea at phenotypic level

\begin{tabular}{|l|l|l|l|l|l|l|l|l|l|l|}
\hline S.No. & Character & $\begin{array}{l}\text { Days to } \\
50 \% \\
\text { Flowering }\end{array}$ & $\begin{array}{l}\text { Plant } \\
\text { Height }\end{array}$ & $\begin{array}{l}\text { Primary } \\
\text { Branches/ } \\
\text { Plant }\end{array}$ & $\begin{array}{l}\text { Pods/ } \\
\text { Plant }\end{array}$ & $\begin{array}{l}\text { Days to } \\
\text { Maturity }\end{array}$ & $\begin{array}{l}\text { Biological } \\
\text { Yield }\end{array}$ & $\begin{array}{l}\text { Harvest } \\
\text { Index }\end{array}$ & $\begin{array}{l}\text { Index } \\
\text { Yield/ } \\
\text { Plant }\end{array}$ \\
\hline $\mathbf{1}$ & Days to 50\% Flowering & $\mathbf{- 0 . 0 0 7 3}$ & 0.0022 & -0.0018 & 0.0008 & -0.0017 & -0.0003 & -0.0012 & -0.0004 & 0.0686 \\
\hline $\mathbf{2}$ & Plant Height & 0.0056 & $\mathbf{- 0 . 0 1 8 4}$ & 0.0056 & 0.0040 & 0.0050 & 0.0050 & 0.0066 & 0.0045 & $-0.3888^{* *}$ \\
\hline $\mathbf{3}$ & Primary Branches/ Plant & -0.0043 & 0.0055 & $\mathbf{- 0 . 0 1 7 9}$ & -0.0004 & -0.0037 & -0.0027 & -0.0003 & -0.0025 & 0.1310 \\
\hline $\mathbf{4}$ & Pods/ Plant & -0.0065 & -0.0138 & 0.0013 & $\mathbf{0 . 0 6 2 6}$ & -0.0052 & 0.0324 & -0.0012 & 0.0151 & $0.5535 * *$ \\
\hline $\mathbf{5}$ & Days to Maturity & -0.0046 & 0.0053 & -0.0040 & 0.0016 & $-\mathbf{0 . 0 1 9 4}$ & 0.0016 & -0.0023 & -0.0034 & -0.0644 \\
\hline $\mathbf{6}$ & Biological Yield & 0.0340 & $-0.2555^{*}$ & 0.1418 & 0.4892 & -0.0770 & $\mathbf{0 . 9 4 5 6 * *}$ & -0.2197 & $0.4591^{* *}$ & $0.9126^{* *}$ \\
\hline $\mathbf{7}$ & Harvest Index & 0.0513 & -0.1124 & 0.0050 & -0.0061 & 0.0364 & -0.0726 & $\mathbf{0 . 3 1 2 5} * *$ & 0.0383 & 0.0953 \\
\hline $\mathbf{8}$ & Seed Index & 0.0004 & -0.0018 & 0.0010 & 0.0018 & 0.0013 & 0.0036 & 0.0009 & $\mathbf{0 . 0 0 7 4}$ & $0.5181 * *$ \\
\hline
\end{tabular}


Correlation coefficient analysis revealed that grain yield exhibited significant and positive correlation both at genotypic and phenotypic level with biological yield ( $\mathrm{rg}=0.96, \mathrm{rp}=0.91)$, pods per plant $(\mathrm{rg}=0.80, \mathrm{rp}=0.0 .55)$ seed index $(\mathrm{rg}=0.0 .82, \mathrm{rp}=0.51)$. Hence direct selection for these traits could be help full in the improvement of chickpea breeding. The perusal of path analysis indicated that biological yield (0.9673) and (0.9126) at genotypic and phenotypic level respectively has high positive direct effect on seed yield per plant. Thus, the character may serve as effective selection parameters in direct breeding programs for yield improvement in chickpea.

\section{References}

Albayrak S., Sevimay C. S., Tongel O. 2006. Effect of inoculation with Rhizobium on seed yield and yield components of common vetch (Vicia sativa L.). Turkish Journal of Agriculture and Forestry. 30: 31-37.0

Al-Jibouri, H.A., P.A. Miller and H.F. Robinson 1958. genotypic and environmental variance in an upland cotton cross of interspecific origion. Agron. J., 50: 633-637.
Arumuganathan, K. and Earle, E.D., Nuclear DNA content of some important species, plant Mol. Biol Rept., 9, 208,1991.

Dewey, D.R. and K.N. Lu (1959). A correlation and path coefficient analysis of component of crested wheat grass seed production. Agron J. 51:515-518.

Johnson, H.W., H.F. Robinson and R.E. Comstock (1955). Genotypic and phenotypic correlation in soyabeans and their implication in selection. Agron. J., 47:477-483.

Robinson, H.F., R. E. Comstock and P.H. Harvey (1951). Genotypic and phenotypic correlation`s in wheat and their implications in selection. Agron J., 43: 282-287.

Thakur, S.R. and Saini, J.P. 1995. Variation, association and path analysis in finger millet (Eleusina coracana) under aerial moisture stress condition. Journal of Agricultural Sciences. 65(1):54-57.

Van der Maesen, L.J.G., Origin, history and taxonomy of chickpea. In The Chickpea, Saxena, M.C. and Singh, K.B., Eds., CAB International, Cambridge, U.K., 11, 1987.

\section{How to cite this article:}

Anmol Kerketta, G.R. Lavanya and Promish Kumar. 2018. Interrelationship between Seed Yield and Component Characters in Chickpea (Cicer arietinum L.). Int.J.Curr.Microbiol.App.Sci. 7(07): 102-106. doi: https://doi.org/10.20546/ijcmas.2018.707.012 